\title{
Giant planets around two intermediate-mass evolved stars and confirmation of the planetary nature of HIP $67851 c^{\star, \star \star, \star \star \star}$
}

\author{
M. I. Jones ${ }^{1}$, J. S. Jenkins², P. Rojo ${ }^{2}$, F. Olivares ${ }^{3}$, and C. H. F. Melo ${ }^{4}$
}

\author{
${ }^{1}$ Department of Electrical Engineering and Center of Astro-Engineering UC, Pontificia Universidad Católica de Chile, \\ Av. Vicuña Mackenna 4860, 782-0436 Macul, Santiago, Chile \\ e-mail:mjones@aiuc.puc.cl \\ 2 Departamento de Astronomía, Universidad de Chile, Camino El Observatorio 1515, Las Condes, Santiago, Chile \\ 3 Departamento de Ciencias Fisicas, Universidad Andres Bello, Av. Republica, Santiago, Chile \\ ${ }^{4}$ European Southern Observatory, Casilla 19001, Santiago 19, Chile
}

Received 9 February 2015 / Accepted 3 June 2015

\section{ABSTRACT}

\begin{abstract}
Context. Precision radial velocities are required to discover and characterize exoplanets. Optical spectra that exhibit many hundreds of absorption lines can allow the $\mathrm{m} \mathrm{s}^{-1}$ precision levels required for this work. After the main-sequence, intermediate-mass stars expand and rotate more slowly than their progenitors, thus, thousands of spectral lines appear in the optical region, permitting the search for Doppler signals in these types of stars.

Aims. In 2009, we began the EXPRESS program, aimed at detecting substellar objects around evolved stars, and studying the effects of the mass and evolution of the host star on their orbital and physical properties.

Methods. We obtained precision radial velocity measurements for the giant stars HIP 65891 and HIP 107773, from CHIRON and FEROS spectra. Also, we obtained new radial velocity epochs for the star HIP 67851, which is known to host a planetary system. Results. We present the discovery of two giant planets around the intermediate-mass evolved star HIP 65891 and HIP 107773 . The best Keplerian fit to the HIP 65891 and HIP 107773 radial velocities leads to the following orbital parameters: $P=1084.5 \mathrm{~d}$; $m_{b} \sin i=6.0 M_{\mathrm{J}} ; e=0.13$ and $P=144.3 \mathrm{~d} ; m_{b} \sin i=2.0 M_{\mathrm{J}} ; e=0.09$, respectively. In addition, we confirm the planetary nature of the outer object orbiting the giant star HIP 67851. The orbital parameters of HIP $67851 c$ are: $P=2131.8 \mathrm{~d}, m_{c} \sin i=6.0 M_{\mathrm{J}}$, and $e=0.17$.

Conclusions. With masses of $2.5 M_{\odot}$ and $2.4 M_{\odot}$, HIP 65891 and HIP 107773 are two of the most massive planet-hosting stars. Additionally, HIP 67851 is one of five giant stars that are known to host a planetary system having a close-in planet $(a<0.7$ AU). Based on the evolutionary states of those five stars, we conclude that close-in planets do exist in multiple systems around subgiants and slightly evolved giants stars, but most likely they are subsequently destroyed by the stellar envelope during the ascent of the red giant branch phase.
\end{abstract}

Key words. techniques: radial velocities - planets and satellites: gaseous planets

\section{Introduction}

Since the discovery of the first planetary system around the pulsar PSR 1257+12 (Wolszczan \& Frail 1992) and the Jupitermass companion to the solar-type star 51 Pegasi (Mayor \& Queloz 1995), the exoplanet field has experienced an exponential growth, leading to the discovery of $\sim 1200$ systems $^{1}$ and more than 3000 unconfirmed candidates from the Kepler mission (Borucki et al. 2010). These planetary systems have been found in very different environments and configurations, showing us that planetary formation is a common phenomenon in our galaxy.

* Based on observations collected at La Silla - Paranal Observatory under programs ID's 085.C-0557, 087.C.0476, 089.C-0524, 090.C0345 and through the Chilean Telescope Time under programs ID's CN 12A-073, CN 12B-047, CN 13A-111, CN-14A-128, and CN-15A-48.

$\star \star$ Appendix $\mathrm{A}$ is available in electronic form at http: //www . aanda.org

$\star \star \star$ RV Tables are only available at the CDS via anonymous ftp to cdsarc.u-strasbg.fr (130.79.128.5) or via

http://cdsarc.u-strasbg.fr/viz-bin/qcat?J/A+A/580/A14

1 http://exoplanet.eu
Although only a small fraction of exoplanets have been found around intermediate-mass stars (IMS; $M_{\star} \gtrsim 1.5 M_{\odot}$ ), they are of great importance, since they allow us to understand the role of stellar mass on the orbital properties and formation efficiency, and to test the validity of planet formation models (e.g. Currie 2009)

Bowler et al. (2010) investigated the period-mass distribution of planets orbiting IMS with $M_{\star} \sim 1.5-2.0 M_{\odot}$, from a uniform sample of 31 subgiants observed by the Lick program (Johnson et al. 2006). They found that their properties are different compared to solar-type host stars at the $4 \sigma$ level. Moreover, they found that the fraction of planets orbiting those stars is $\sim 26 \%$, compared to only $\sim 10 \%$ for solar-type hosts. In addition, based on a much larger sample of 1266 stars observed by the California Planet Survey (Howard et al. 2010), Johnson et al. (2010) showed that there is a linear increase in the fraction of planets, from $f=0.03$ to $f=0.14$, in the mass range between $\sim 0.5-2.0 M_{\odot}$. These observational results tell us that planet formation efficiency is strongly dependent on the stellar mass, however, the reliability of the derived masses of subgiant host stars has recently been called into question. Lloyd $(2011,2013)$ showed that the mass distribution of the planethosting subgiants is incompatible with the distribution derived 
from integrating isochrones, concluding that these stars have masses of $\sim 1.0-1.2 M_{\odot}$. Similarly, based on Galactic kinematics, Schlaufman \& Winn (2013) concluded that the subgiant host stars are similar in mass to solar-type host stars. If this is the case, however, then the planetary systems around them should exhibit the same orbital properties and detection fraction as for planets around less massive stars.

Finally, based on a sample of 373 giant stars targeted by the Lick radial velocity (RV) survey (Frink et al. 2002), Reffert et al. (2015) studied the occurrence rate of planets around stars with masses between $\sim 1.0-3.0 M_{\odot}$. They showed that the fraction of exoplanets increases with increasing stellar mass, with a peak at $\sim 1.9 M_{\odot}$, and that there is a rapid drop in the occurrence rate for stars more massive than $\sim 2.5 M_{\odot}$.

In this paper, we present the discovery of two planets orbiting the intermediate-mass giant stars HIP 65891 and HIP 107773. These are two of the most massive stars that are known to host substellar companions. HIP $65891 b$ and HIP $107773 b$ are the sixth and seventh substellar objects discovered by the EXPRESS (EXoPlanets aRound Evolved StarS) survey (Jones et al. 2011, 2015). Additionally, we present new RV epochs of the giant star HIP 67851. These velocities allowed us to confirm the planetary nature of the outer object in the system, as suggested by Jones et al. (2015). The paper is organized as follows. In Sect. 2, we briefly present the observations, data reduction, and the calculation methods we used to obtain the radial velocities. In Sect. 3, we summarize the properties of the host stars. In Sect. 4, we present the orbital parameters of HIP $65891 b$, HIP $107773 b$ and HIP 67851c, as well as improved orbital parameters for HIP $67851 b$. In Sect. 5 the stellar activity analysis is presented. Finally, in Sect. 6, we present the summary and discussion.

\section{Observations and data reduction}

We collected a total of 26 spectra of HIP 65891 and 36 spectra of HIP 107773 using CHIRON (Tokovinin et al. 2013), a high-resolution stable spectrograph installed in the $1.5 \mathrm{~m}$ telescope at Cerro Tololo Inter-American Observatory. Using the image slicer mode $(R \sim 80000)$, we typically obtained a signal-to-noise-ratio $(\mathrm{S} / \mathrm{N})$ of $\sim 100$ with $400 \mathrm{~s}$ of integration for HIP 65891 and $\sim 150 \mathrm{~s}$ for HIP 107773. The data reduction was performed using the CHIRON data reduction system. The pipeline does a standard echelle reduction, i.e., bias subtraction, flat-field correction, order tracing, extraction, and wavelength calibration. Additionally, we use an iodine cell in the "IN" position, meaning that it is placed in the light path, at the fiber exit. The cell contains molecular iodine $\left(\mathrm{I}_{2}\right)$, which superimposes a forest of absorption lines in the region between 5000-6000 $\AA$. These lines are used as precise wavelength markers against which the doppler shift of the stellar spectrum is measured. The radial velocity variations were calculated according to the method described in Butler et al. (1996) and Jones et al. (2014, 2015). We achieve a mean RV precision of $\sim 5 \mathrm{~m} \mathrm{~s}^{-1}$ from CHIRON spectra using this method.

In addition, we took 24 spectra of HIP 65891 and 27 spectra of HIP 107773 using the Fiber-fed Extended Range Optical Spectrograph (FEROS; Kaufer et al. 1999) mounted at the $2.2 \mathrm{~m}$ telescope at La Silla Observatory. We discarded one FEROS spectrum of HIP 65891 since there was a problem with a folding mirror in the calibration unit. The typical observing time was $\sim 60 \mathrm{~s}$ and $\sim 180 \mathrm{~s}$ (for HIP 65891 and HIP 107773, respectively), leading to a $S / N \sim 100$ per pixel. The data reduction of the spectra was performed with the FEROS pipeline. The radial velocities were computed using the simultaneous calibration
Table 1. Stellar properties.

\begin{tabular}{lrrr}
\hline \hline & HIP 65891 & HIP 107773 & HIP 67851 \\
\hline Spectral type & K0III & K1III & K0III \\
$B-V(\mathrm{mag})$ & 1.00 & 1.02 & 1.01 \\
$V$ (mag) & 6.75 & 5.62 & 6.17 \\
Parallax (mas) & $7.35 \pm 0.60$ & $9.65 \pm 0.40$ & $15.16 \pm 0.39$ \\
$T_{\text {eff }}(\mathrm{K})$ & $5000 \pm 100$ & $4945 \pm 100$ & $4890 \pm 100$ \\
$L\left(L_{\odot}\right)$ & $44.8 \pm 9.6$ & $74.0 \pm 9.2$ & $17.55 \pm 2.64$ \\
$\log g\left(\mathrm{~cm} \mathrm{~s}^{-2}\right)$ & $2.9 \pm 0.2$ & $2.6 \pm 0.2$ & $3.2 \pm 0.2$ \\
{$[\mathrm{Fe} / \mathrm{H}]\left(\mathrm{dex}^{-1}\right.$} & $0.16 \pm 0.10$ & $0.03 \pm 0.10$ & $0.00 \pm 0.10$ \\
$v \sin i\left(\mathrm{~km} \mathrm{~s}^{-1}\right)$ & $2.5 \pm 0.9$ & $2.0 \pm 0.9$ & $1.8 \pm 0.9$ \\
$M_{\star}\left(M_{\odot}\right)$ & $2.50 \pm 0.21$ & $2.42 \pm 0.27$ & $1.63 \pm 0.22$ \\
$R_{\star}\left(R_{\odot}\right)$ & $8.93 \pm 1.02$ & $11.6 \pm 1.4$ & $5.92 \pm 0.44$ \\
\hline
\end{tabular}

method (Baranne et al. 1996), according to the method described in Jones et al. (2013) and Jones \& Jenkins (2014).

\section{Stellar properties}

The stellar parameters of HIP 65891 and HIP 107773 are summarized in Table 1 . The spectral types, $V$ magnitudes, $B-V$ colors, and parallaxes were taken from the HIPPARCOS cata$\log$ (Van Leeuwen 2007). The atmospheric parameters were retrieved from Jones et al. (2011). For each star we created 100 synthetic data sets for $T_{\text {eff }}, \log L$, and [Fe/H], assuming Gaussian distributed errors. Then we compared these synthetic data sets with Salasnich et al. (2000; S00 hereafter) models, following the method presented in Jones et al. (2011). The resulting values for $M_{\star}$ and $R_{\star}$ correspond to the mean and the root mean square (RMS) of the two resulting distributions.

Figure 1 shows a HR diagram with the positions of HIP 65891 (open square) and HIP 107773 (filled circle). For comparison, two S00 models with solar metallicity are overplotted. As can be seen, HIP 65891 is most likely at the base of the red giant branch (RGB) phase, since no horizontal branch (HB) model intersects its position. The small panel shows a zoomed region of the HIP 107773 position and its closest evolutionary track in the grid $\left(M_{\star}=2.5 M_{\odot}\right.$ and $\left.[\mathrm{Fe} / \mathrm{H}]=0.0\right)$. The blue solid and red dashed lines correspond to the RGB and HB phase, respectively. The dots are the points in the grid. As can be seen, it is not clear whether the star is ascending the RGB or has already reached the He-core burning phase. However, according to these evolutionary models, the timescales between point $\mathrm{A}$ and B is 200 times shorter than between C and D. Therefore, based on the ratio of these timescales, we conclude that HIP 107773 is most likely a HB star.

The stellar properties of HIP 67851 (retrieved from Jones et al. 2015) are also summarized in Table 1.

\section{Orbital parameters}

\subsection{HIP $65891 b$}

The RVs of HIP 65891 are listed in Tables A.1 and A.2. A Lomb-Scargle (LS) periodogram (Scargle 1982) of the data revealed a strong peak at $\sim 1019$ days with a false alarm probability (FAP) of $\sim 10^{-7}$. Starting from this period, we use the Systemic Console 2.17 (Meschiari et al. 2009) and we obtained a single-planet solution with the following orbital parameters: $P=1084.5 \pm 23.2 \mathrm{~d}, K=64.9 \pm 2.4 \mathrm{~m} \mathrm{~s}^{-1}$ (corresponding to $m_{b} \sin i=6.0 \pm 0.49 M_{\mathrm{J}}$ ), and $e=0.13 \pm 0.05$. The uncertainties were obtained using the bootstrap tool provided by the 
Table 2. Orbital parameters.

\begin{tabular}{lrrrr}
\hline \hline & HIP 65891 $b$ & HIP 107773b & HIP 67851 $b$ & HIP 67851 $c$ \\
\hline$P($ days $)$ & $1084.5 \pm 23.2$ & $144.3 \pm 0.5$ & $88.9 \pm 0.1$ & $2131.8 \pm 88.3$ \\
$K\left(\mathrm{~m} \mathrm{~s}^{-1}\right)$ & $64.9 \pm 2.4$ & $42.7 \pm 2.7$ & $45.5 \pm 1.6$ & $69.0 \pm 3.3$ \\
$a(\mathrm{AU})$ & $2.81 \pm 0.09$ & $0.72 \pm 0.03$ & $0.46 \pm 0.02$ & $3.82 \pm 0.23$ \\
$e$ & $0.13 \pm 0.05$ & $0.09 \pm 0.06$ & $0.05 \pm 0.04$ & $0.17 \pm 0.06$ \\
$m \sin i\left(M_{\mathrm{J}}\right)$ & $6.00 \pm 0.49$ & $1.98 \pm 0.21$ & $1.38 \pm 0.15$ & $5.98 \pm 0.76$ \\
$\omega(\mathrm{deg})$ & $355.5 \pm 15.5$ & $166.0 \pm 32.6$ & $138.1 \pm 60.0$ & $166.5 \pm 20.5$ \\
$T_{\mathrm{P}}-2450000$ & $6014.8 \pm 49.3$ & $6202.3 \pm 12.8$ & $2997.8 \pm 16.7$ & $2684.1 \pm 235.7$ \\
$\gamma_{1}\left(\mathrm{~m} \mathrm{~s}^{-1}\right)(\mathrm{CHIRON})$ & $7.9 \pm 4.7$ & $-18.4 \pm 4.6$ & $29.0 \pm 3.8$ & $29.0 \pm 3.8$ \\
$\gamma_{2}\left(\mathrm{~m} \mathrm{~s}^{-1}\right)($ FEROS $)$ & $2.5 \pm 3.2$ & $14.1 \pm 2.5$ & $-31.5 \pm 4.3$ & $-31.5 \pm 4.3$ \\
$\dot{\gamma}\left(\mathrm{m} \mathrm{s}^{-1} \mathrm{yr}^{-} 1\right)$ & - & $14.6 \pm 2.5$ & - & - \\
$\mathrm{rms}\left(\mathrm{m} \mathrm{s}^{-1}\right)$ & 9.3 & 12.0 & 8.9 & 8.9 \\
$\chi_{\text {red }}^{2}$ & 3.5 & 5.7 & 3.1 & 3.1 \\
\hline
\end{tabular}

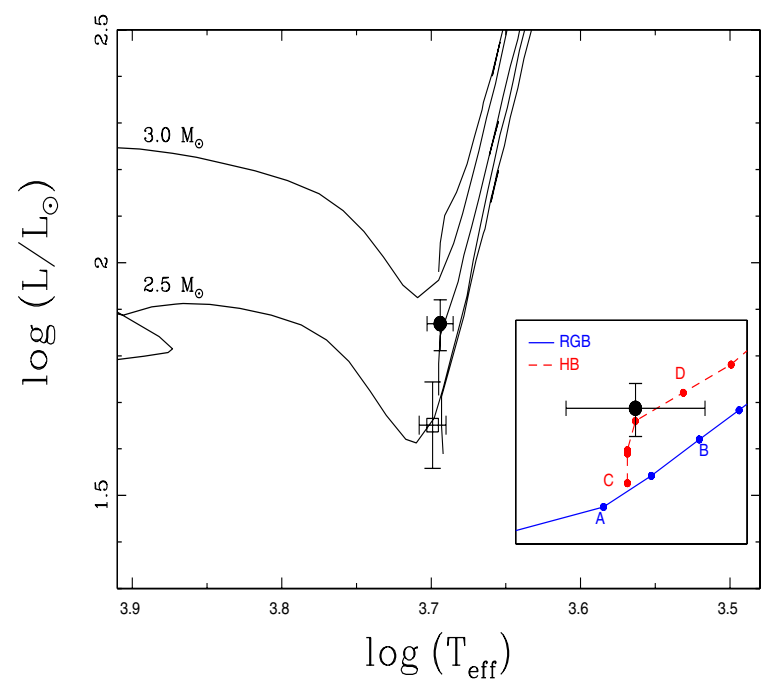

Fig. 1. Position of HIP 65891 (open square) and HIP 107773 (filled circle) in the HR diagram. Different S00 evolutionary tracks with solar metallicity are overplotted. The small panel shows a zoomed region of the HIP 107773 position and its closest isomass track in the grid.

Systemic Console. The uncertainties in the semimajor axis and planet mass were computed by error propagation of these values, also including the uncertainty in the stellar mass ${ }^{2}$. The full set of orbital parameters are listed in Table 2. Figure 2 shows the radial velocity curve. The red triangles and black circles correspond to CHIRON and FEROS data, respectively. The Keplerian fit is overplotted (solid line), leading to a rms of $9.3 \mathrm{~m} \mathrm{~s}^{-1}$.

\subsection{HIP $107773 b$}

The radial velocity measurements of HIP 107773 computed from CHIRON and FEROS spectra are listed in Tables A.3 and A.4, respectively. A periodogram analysis of the data revealed a 144.3-days signal with a FAP of $\sim 10^{-7}$. The best Keplerian fit of the data leads to a one-planet system plus a linear trend with the following parameters: $P=144.3 \pm 0.5 \mathrm{~d}$, $K=42.7 \pm 2.7 \mathrm{~m} \mathrm{~s}^{-1}$ (corresponding to $m_{b} \sin i=2.0 \pm 0.2 M_{\mathrm{J}}$ ), $e=0.09 \pm 0.06$, and $\dot{\gamma}=14.6 \pm 2.5 \mathrm{~m} \mathrm{~s}^{-1} \mathrm{yr}^{-1}$. The solution with a linear trend significantly improves the rms of the fit (from

\footnotetext{
2 The Systemic Console does not include the contribution from the uncertainty in the stellar mass.
}

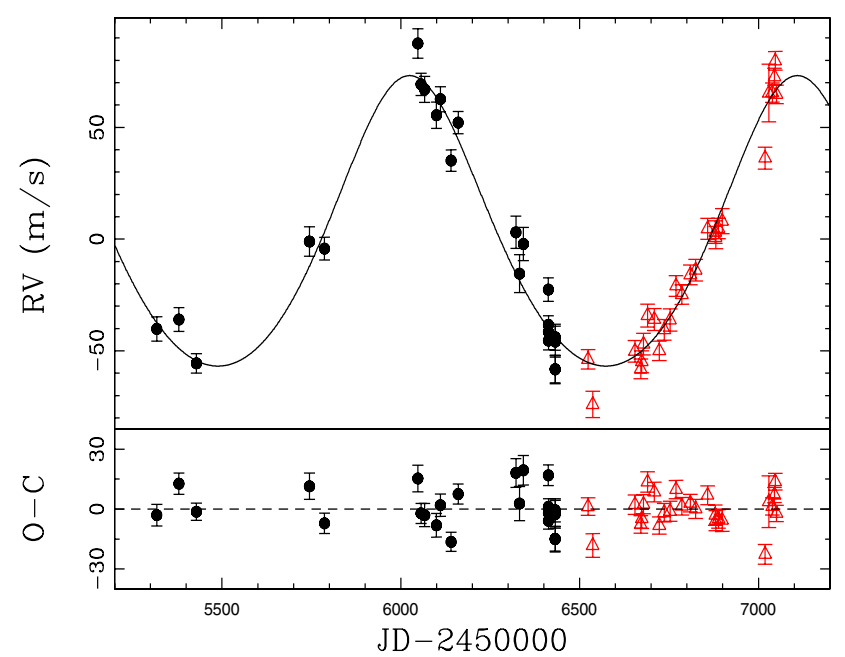

Fig. 2. Upper panel: radial velocity variations of HIP 65891. The red triangles and black circles correspond to CHIRON and FEROS velocities, respectively. The best Keplerian solution is overplotted (black solid line). Lower panel: residuals from the Keplerian fit. The rms of the fit is $9.3 \mathrm{~m} \mathrm{~s}^{-1}$.

$17.8 \mathrm{~m} \mathrm{~s}^{-1}$ to $12.0 \mathrm{~m} \mathrm{~s}^{-1}$ ). The $F$ value computed from the ratio of the $\chi_{\text {red }}^{2}$ with seven and eight parameters is 2.1. The probability of exceeding such value (assuming an $F$-distribution; see Bevington \& Robinson 2003) is 0.16. We derived the minimum mass and orbital distance of the second planet in the system of $m_{c} \gtrsim 2.8 M_{\mathrm{J}}$ and $a_{c} \gtrsim 5.9$ AU from $\dot{\gamma}$, using the relation given in Winn et al. (2009). The orbital parameters are also listed in Table 2. Figure 3 shows the RV measurements from CHIRON and FEROS spectra (red triangles and black circles, respectively) and the Keplerian fit (solid black line). The rms of the post-fit residuals is $11.9 \mathrm{~m} \mathrm{~s}^{-1}$.

\subsection{HIP $67851 c$}

We obtained new CHIRON RV epochs, which allowed us to better sampling the orbital period of HIP $67851 c$, and thus to confirm its planetary nature, as proposed by Jones et al. (2015). In addition, we found two FEROS spectra of HIP67851 in the ESO archive, which were taken in 2004. Figure 4 shows the RV curve of HIP 67851. The red triangles and black circles correspond to CHIRON and FEROS data, respectively. The best two-planets solution is overplotted (black solid line). The 


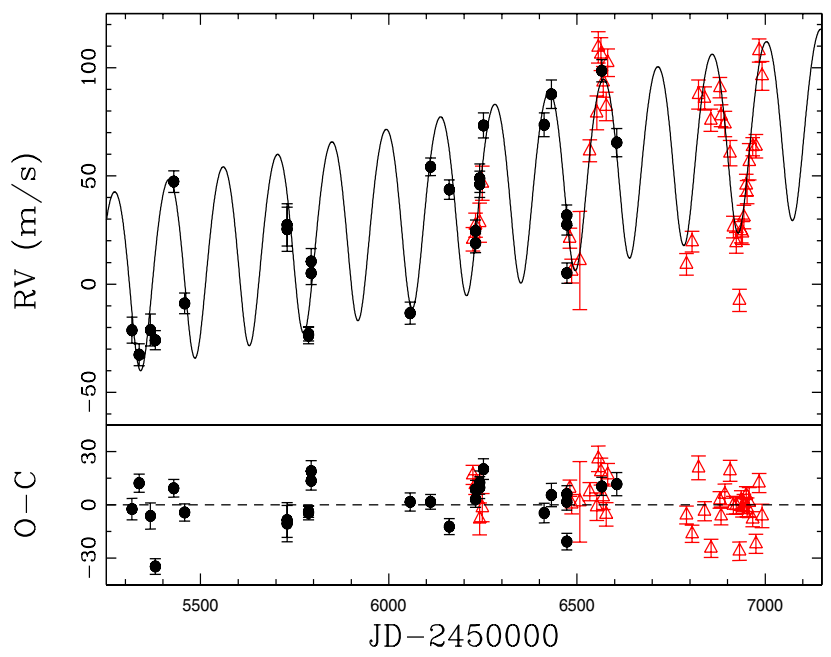

Fig. 3. Upper panel: radial velocities measurements of HIP 107773. The red triangles and black circles correspond to CHIRON and FEROS velocities, respectively. The best Keplerian solution, including a linear trend, is overplotted (black solid line). Lower panel: residuals from the Keplerian fit. The rms around the fit is $11.9 \mathrm{~m} \mathrm{~s}^{-1}$.

orbital parameters of HIP 67851 $c$ are: $P=2131.8 \pm 88.3 \mathrm{~d}$, $K=69.0 \pm 3.3 \mathrm{~m} \mathrm{~s}^{-1}$ (corresponding to $m_{c} \sin i=6.0 \pm 0.8 M_{\mathrm{J}}$ ), and $e=0.17 \pm 0.06$. These values, as well as the refined orbital parameters of HIP $67851 b$, are listed in Table 2. We note that Wittenmyer et al. (2015) recently presented RV measurements of HIP 67851 from the Pan-Pacific Planet Search (PPPS; Wittenmyer et al. 2011). They recovered the signal of HIP $67851 b$ and confirmed the presence of an outer planet in the system. However, they obtained an orbital period of $1626 \pm 26 d$ and minimum mass of the planet of $3.6 \pm 0.6 M_{\mathrm{J}}$ (assuming a stellar mass of $1.3 M_{\odot}$ ), which is incompatible with our solution. The reason for this discrepancy is because their orbital solution of HIP 67851 $c$ relies on one RV data-point, which is most likely an outlier. In fact, a new reduction of the PPPS data set, including new RV epochs, is soon to be published, and the new solution is in good agreement with the solution presented here (Wittenmyer; priv. comm.).

\section{Intrinsic stellar phenomena}

To test the nature of the RV signals detected in HIP 65891 and HIP 107773 , we analyzed the HIPPARCos photometric data of these two stars. The HIP 65891 data set is comprised of 123 high quality epochs, spanning 3.2 years. The photometric variability is less than $0.01 \mathrm{mag}$ and a periodogram analysis revealed no significant periodicity in the data. Similarly, the photometric data of HIP 107773 is comprised of 221 high-quality measurements, with a baseline of 3.2 years. This data set revealed a photometric variability of $\sigma=0.007 \mathrm{mag}$, and no periodicity is observed in a LS periodogram. Also, from the projected rotational velocity and the stellar radius, we can put an upper limit on the stellar rotational period. In the case of HIP 65891, we obtained a value of $179 \mathrm{~d}$, meaning that we can discard the hypothesis that the observed RV variation is related to the rotation of star. In the case of HIP 107773, we computed a maximum rotational period of $293 \mathrm{~d}$, which is more than two times larger than RV period. An inclination angle of $i \sim 29$ degrees is required for the rotational period to match the RV period.
In additional, we analyzed the line profile variations by computing the bisector velocity span (BVS) and full-width at half maximum (FWHM) variations of the cross-correlation function (CCF), in a similar way to that presented in Jones et al. (2014). Also, we computed the chromospheric activity indexes from integrating the flux in the core of the Ca II HK lines, in the same manner as described in Jenkins et al. (2008, 2011). We only used FEROS spectra since CHIRON does not cover the spectral region where these lines are located. These results are shown in Figs. 5 and 6. The Pearson correlation coefficients are also labeled. Clearly there is no significant correlation between these quantities and the radial velocities of any of the two stars. Also, a LS periodogram analysis revelead no significant peak in these quantities, with the exception of a peak (FAP $\sim 0.009)$ in the FWHM variations of HIP 107773, around $\sim 183 \mathrm{~d}$. This value is significantly longer than the 144-day period observed in the RV data.

Lastly, most giant stars bluer than $B-V<1.2$ exhibit pulsation-induced RV variability at the $10-20 \mathrm{~m} \mathrm{~s}^{-1}$ (Sato et al. 2005; Hekker et al. 2006), which is well below the amplitudes observed in these stars. In fact, the Kjeldsen \& Bedding (1995) scaling relations predict RV amplitudes of $\sim 4$ and $7 \mathrm{~m} \mathrm{~s}^{-1}$ for HIP 65891 and HIP 107773, respectively. The corresponding lifetimes of the maximum power oscillations $\left(1 / v_{\max }\right)$ are 2.7 and $4.7 \mathrm{~h}$, respectively.

\section{Summary and discussion}

We present precision radial velocities for the giant stars HIP 65891 and HIP 107773. The two data sets revealed periodic signals, which are most likely explained by the presence of giant planets orbiting these two stars. The best Keplerian fit to the HIP 65891 data leads to the following orbital parameters: $P=1084.5 \pm 23.2 \mathrm{~d}, m_{b} \sin i=6.0 \pm 0.5 M_{\mathrm{J}}$, and $e=$ $0.13 \pm 0.05$. Similarly, the orbital solution for HIP $107773 b$ is: $P=144.3 \mathrm{~d} \pm 0.5, m_{b} \sin i=2.0 \pm 0.2 M_{\mathrm{J}}$, and $e=0.09 \pm 0.06$, plus a linear trend of $\dot{\gamma}=14.6 \pm 2.5 \mathrm{~m} \mathrm{~s}^{-1} \mathrm{yr}^{-1}$. We derived a mass of $2.5 M_{\odot}$ and $2.4 M_{\odot}$ for HIP 65891 and HIP 107773, respectively, meaning that they are amongst the most massive stars that are known to host planets. Although there relatively few known planets around stars more massive than $\sim 2.0 M_{\odot}$, they are fairly common and are of great interest, since they allow us to understand the role of the stellar mass in the formation and characteristics of planetary systems.

So far, we have discovered seven substellar objects around six giant stars. Interestingly, all of these host stars are IMSs, despite the fact that $\sim 25 \%$ of our targets are low-mass stars. This result confirms that the frequency of planets increases with the stellar mass (e.g., Bowler et al. 2010; Johnson et al. 2010; Reffert et al. 2015). A detailed statistical analysis of the occurrence rate from the EXPRESS program will be presented soon (Jones et al., in prep.).

Finally, we present new radial velocity epochs of the giant star HIP 67851, confirming the presence of an outer planet in the system. We obtained the following orbital parameter for HIP 67851c: $P=2131.8 \pm 88.3 \mathrm{~d}, m_{c} \sin i=6.0 \pm$ $0.8 M_{\mathrm{J}}$, and $e=0.17 \pm 0.06$. Apart from HIP 67851, there are eight giant stars $(\log g \lesssim 3.6)$ that are known to host multiplanet systems ${ }^{3}$ : namely, 24 Sextantis and HD 200964 (Johnson et al. 2011), HD 4732 (Sato et al. 2013), Kepler 56

3 The outer planet around HD 47536 (Setiawan et al. 2008) was shown likely not to be real (Soto et al. 2015). Also, the proposed system around BD+202457 (Niedzielski et al. 2009) was shown to be dynamically unstable (see Horner et al. 2014). 
M. I. Jones et al.: Giant planets around two intermediate-mass evolved stars

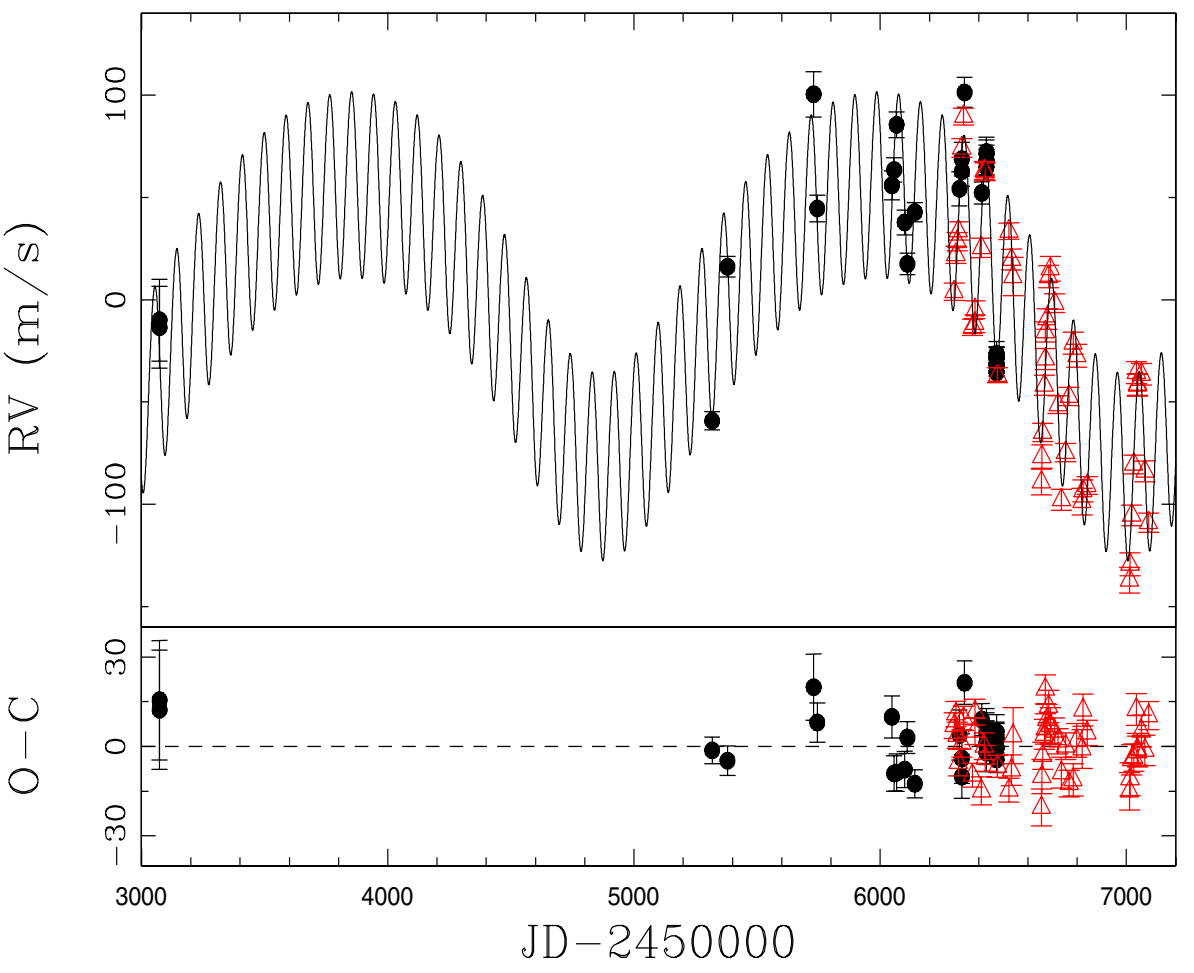

Fig. 4. Upper panel: radial velocity variations of HIP 67851. The red triangles and black circles correspond to CHIRON and FEROS velocities, respectively. The best two-planet solution is overplotted (black solid line). Lower panel: residuals from the Keplerian fit. The rms around the fit is $8.9 \mathrm{~m} \mathrm{~s}^{-1}$.
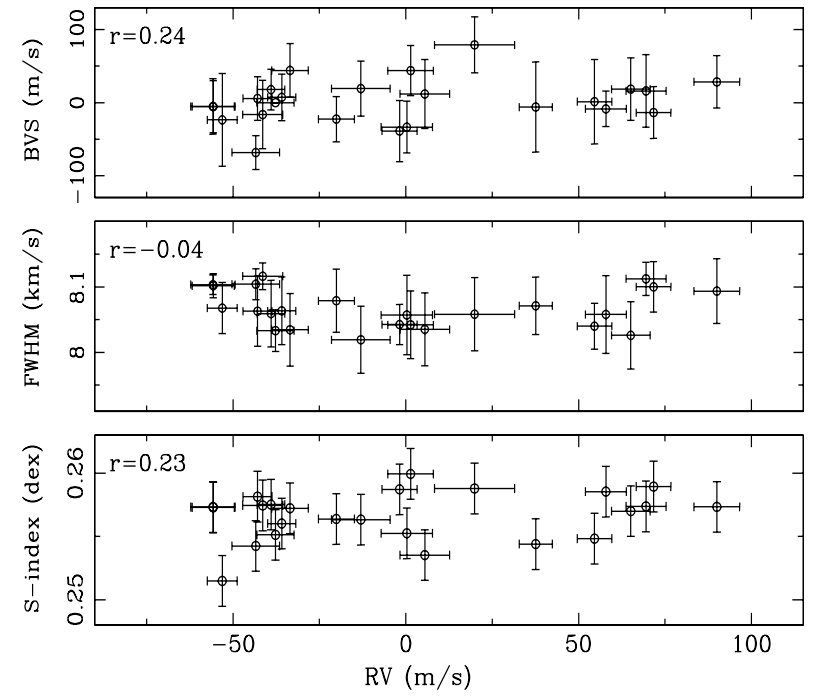

Fig. 5. BVS, FWHM, and S-index variations versus FEROS radial velocities of HIP 65891 (upper, middle, and lower panel, respectively).

(Huber et al. 2013), Kepler 432 (Ciceri et al. 2015; Ortiz et al. 2015; Quinn et al. 2015), Kepler 391 (Rowe et al. 2014), $\eta$ Ceti (Trifonov et al. 2014), and TYC 1422-614-1 (Niedzielski et al. 2015). Interestingly, $\eta$ Ceti is the only one that is located in the clump region, although according to Trifonov et al. (2014) it is most likely a RGB star instead of a HB star. The rest of them are very close to the base of the RGB phase, therefore they still have relatively small radii. Moreover, Kepler $56 b$, Kepler $391 b$, Kepler 432 b, HIP 67851 $b$, and TYC-1422-614-1 $b$ are located in close-in orbits $(a<0.7 \mathrm{AU})$, a region where planets are rare around post-MS stars. This observational result suggests that systems with short period planets do exist around slightly evolved stars, but they are destroyed by the stellar envelope during the late stage of the RGB phase, when the stellar radius grows sufficiently large $\left(R_{\star} \sim a\right)$. This could explain why these
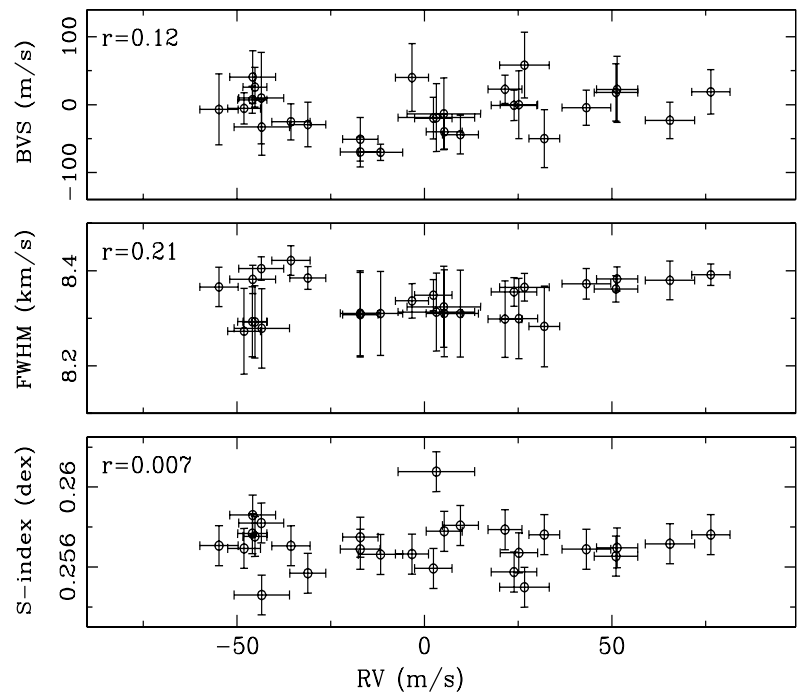

Fig. 6. BVS, FWHM, and S-index variations versus FEROS radial velocities of HIP 107773 (upper, middle, and lower panel, respectively).

kind of systems are not found around HB giants and therefore why close-in planets are not found around these evolved stars. However, the stellar mass might play an important role on shaping the inner regions of planetary systems, particularly since the aforementioned stars are on average less massive than the bulk of planet-hosting giant stars.

Acknowledgements. M.J. acknowledges financial support from Fondecyt project \#3140607 and FONDEF project CA13I10203. J.J. and P.R. acknowledge funding by the CATA-Basal grant (PB06, Conicyt). P.R. acknowledges support from Fondecyt project \#1120299. F.O acknowledges financial support from Fondecyt project \#3140326 and by the Ministry of Economy, Development, and Tourism's Millennium Science Initiative through grant IC12009, awarded to The Millennium Institute of Astrophysics, MAS. We acknowledge the anonymous referee for very useful comments, which helped to improve the quality of this work. This research has made use of the SIMBAD database and the VizieR catalogue access tool, operated at CDS, Strasbourg, France. 


\section{References}

Baranne, A., Queloz, D., Mayor, M., et al. 1996, A\&A, 119, 373

Bevington, P. R., \& Robinson, D. K. 2003. Data Reduction and Error Analysis for the Physical Sciences, 3rd edn. (New-York: McGraw-Hill Education)

Borucki, W. J., Koch, D., Basri, G., et al. 2010, Science, 327, 977

Bowler, B. P., Johnson, J. A., Marcy, G. W., et al. 2010, ApJ, 709, 396

Butler, R. P., Marcy, G. W., Williams, E., et al. 1996, PASP, 108, 500

Ciceri, S. Lillo-Box, J., Southworth, J., et al. 2015, A\&A, 573, L5

Currie, T. 2009, ApJ, 694, 171

Frink, S., Mitchell, D. S., Quirrenbach, A., et al. 2002, ApJ, 576, 478

Hekker, S., Reffert, S., Quirrenbach, A., et al. 2006, A\&A, 454, 943

Howard, A. W., Johnson, J. A., Marcy, G. W., et al. 2010, ApJ, 721, 1467

Horner, J., Wittenmyer, R. A., Hinse, T. C., \& Marshall, J. P. 2014, MNRAS,

439,1176

Huber, D., Carter, J. A., Barbieri, M., et al. 2013, Science, 342, 331

Jenkins, J. S., Jones, H. R. A., Pavlenko, Y., et al. 2008, A\&A, 485, 571

Jenkins, J. S., Murgas, F., Rojo, P., et al. 2011, A\&A, 531, A8

Johnson, J. A., Marcy, G. W., Fischer, D. A., et al. 2006, ApJ, 652, 1724

Johnson, J. A., Aller, K. M., Howard, A. W., \& Krupp, J. R. 2010, PASP, 122, 905

Johnson, J. A., Payne, M., Howard, A., et al. 2011, AJ, 141, 16

Jones, M. I., \& Jenkins, J. S. 2014, A\&A, 562, A129

Jones, M. I., Jenkins, J. S., Rojo, P., \& Melo, C. H. F. 2011, A\&A, 536, A71

Jones, M. I., Jenkins, J. S., Rojo, P., Melo, C. H. F., \& Bluhm, P. 2013, A\&A, 556, A78

Jones, M. I., Jenkins, J. S., Bluhm, P., Rojo, P., \& Melo, C. H. F. 2014, A\&A, 566, A113

Jones, M. I., Jenkins, J. S., Rojo, P., Melo, C. H. F., \& Bluhm, P. 2015, A\&A, 573, A3

Kaufer, A., Stahl, O., Tubbesing, S., et al. 1999, The Messenger, 95, 8
Kjeldsen, H., \& Bedding, T. R. 1995, A\&A, 293, 87

Lloyd, J. P. 2011, ApJ, 739, 49

Lloyd, J. P. 2013, ApJ, 774, 2

Mayor, M., \& Queloz, D. 1995, Nature, 378, 355

Meschiari, S., Wolf, A. S., Rivera, E., et al. 2009, PASJ, 121, 1016

Niedzielski, A., Nowak, G., Adamów, M., \& Wolszczan, A. 2009, ApJ, 707, 768

Niedzielski, A., Villaver, E., Adamów, M., et al. 2015, A\&A, 573, A36

Ortiz, M., Gandolfi, D., Reffert, S., et al. 2015, A\&A, 573, L6

Quinn, S. N., White, T. R., Latham, D. W., et al. 2015, ApJ, 803, 49

Reffert, S., Bergmann, C., Quirrenbach, A., et al. 2015, A\&A, 574, A116

Rowe, J. F., Bryson, S. T., Marcy, G. W., et al. 2014, ApJ, 784, 45

Salasnich, B., Girardi, L., Weiss, A., \& Chiosi, C. 2000, A\&A, 361, 1023

Sato, B., Eiji, K., Yoichi, T., et al. 2005, PASJ, 57, 97

Sato, B., Omiya, M., Wittenmyer, R. A., et al. 2013, ApJ, 762, 9

Setiawan, J., Weise, P., Henning, Th., et al. 2008, Precision Spectroscopy in Astrophysics, Proc. ESO/Lisbon/Aveiro Conference held in Aveiro, Portugal, 11-15 September 2006, eds. N. C. Santos, L. Pasquini, A. C. M. Correia, \& M. Romanielleo, Garching, Germany, 201

Scargle, J. D. 1982, ApJ, 263, 835

Schlaufman, K., \& Winn, J. 2013, ApJ, 772, 143

Soto, M., Jenkins, J. S., \& Jones, M. I. 2015, MNRAS, 451, 3131

Tokovinin, A., Fischer, D. A., Bonati, M., et al. 2013, PASP, 125, 1336

Trifonov, T., Reffert, S., Tan, X., Lee M. H., \& Quirrenbach, A. 2014, A\&A, 568, A64

Van Leeuwen, F. 2007, A\&A, 474, 653

Winn, J. N., Johnson, J. A., Albrecht, S., et al. 2009, ApJ, 703, 99

Wittenmyer, R. A., Endl, M., Wang, L., et al. 2011, ApJ, 743, 184

Wittenmyer, R. A., Wang, L., Liu, F., et al. 2015, ApJ, 800, 74

Wolszczan, A., \& Frail, D. A. 1992, Nature, 355, 145

Pages 7 to 8 are available in the electronic edition of the journal at http: //www . aanda.org 
M. I. Jones et al.: Giant planets around two intermediate-mass evolved stars

\section{Appendix A: Radial velocity tables.}

Table A.1. CHIRON radial velocity variations of HIP 65891.

\begin{tabular}{rrr}
\hline \hline JD - 2450 000 & $\begin{array}{r}\mathrm{RV} \\
\left(\mathrm{m} \mathrm{s}^{-1}\right)\end{array}$ & $\begin{array}{r}\text { Error } \\
\left(\mathrm{m} \mathrm{s}^{-1}\right)\end{array}$ \\
\hline 6523.4823 & -45.9 & 4.4 \\
6536.4923 & -66.1 & 5.9 \\
6654.7915 & -42.3 & 4.8 \\
6670.8031 & -50.2 & 4.4 \\
6673.8555 & -47.0 & 4.9 \\
6678.8188 & -39.1 & 4.8 \\
6689.8292 & -26.3 & 5.1 \\
6708.8389 & -28.1 & 4.9 \\
6722.6806 & -42.1 & 4.3 \\
6736.6035 & -32.7 & 4.6 \\
6752.6675 & -28.3 & 5.0 \\
6769.6338 & -13.1 & 4.6 \\
6785.6895 & -16.9 & 4.4 \\
6809.5599 & -8.1 & 4.4 \\
6824.5449 & -6.0 & 4.8 \\
6857.4830 & 12.5 & 4.7 \\
6879.5110 & 11.0 & 5.1 \\
6880.4898 & 8.6 & 5.0 \\
6888.4805 & 12.6 & 4.6 \\
6898.4812 & 15.9 & 5.6 \\
7018.8291 & 44.1 & 4.9 \\
7028.8280 & 73.2 & 12.9 \\
7038.8417 & 73.4 & 4.3 \\
7044.8105 & 80.5 & 3.8 \\
7046.7597 & 87.6 & 4.2 \\
7050.7543 & 72.6 & 4.1 \\
\hline & &
\end{tabular}

Table A.2. FEROS radial velocity variations of HIP 65891.

\begin{tabular}{lrr}
\hline \hline JD - 2450 000 & $\begin{array}{r}\mathrm{RV} \\
\left(\mathrm{m} \mathrm{s}^{-1}\right)\end{array}$ & $\begin{array}{r}\text { Error } \\
\left(\mathrm{m} \mathrm{s}^{-1}\right)\end{array}$ \\
\hline 5317.6274 & -37.7 & 5.4 \\
5379.5439 & -33.5 & 5.3 \\
5428.5059 & -53.1 & 4.3 \\
5744.5713 & 1.4 & 6.6 \\
5786.5649 & -1.8 & 5.1 \\
6047.5977 & 90.0 & 6.6 \\
6056.5850 & 71.7 & 5.0 \\
6066.5972 & 69.5 & 5.8 \\
6099.5825 & 57.9 & 5.9 \\
6110.5620 & 65.1 & 5.6 \\
6140.6284 & 37.6 & 4.8 \\
6160.5493 & 54.6 & 5.0 \\
6321.7749 & 5.5 & 7.2 \\
6331.8145 & -13.0 & 8.5 \\
6342.7432 & 0.3 & 7.4 \\
6412.6270 & -20.1 & 5.2 \\
6412.7261 & -35.9 & 4.1 \\
6412.7520 & -42.9 & 4.2 \\
6412.8042 & -39.0 & 4.0 \\
6431.5796 & -55.8 & 6.4 \\
6431.6436 & -55.7 & 6.1 \\
6431.6802 & -41.4 & 5.8 \\
6431.7441 & -43.4 & 6.9 \\
\hline
\end{tabular}

Table A.3. CHIRON radial velocity variations of HIP 107773.

\begin{tabular}{lrr}
\hline \hline JD - 2450 000 & $\begin{array}{r}\text { RV } \\
\left(\mathrm{m} \mathrm{s}^{-1}\right)\end{array}$ & $\begin{array}{r}\text { Error } \\
\left(\mathrm{m} \mathrm{s}^{-1}\right)\end{array}$ \\
\hline 6223.5346 & -34.0 & 5.4 \\
6230.5344 & -27.5 & 5.6 \\
6241.5757 & -26.3 & 9.0 \\
6248.5202 & -8.1 & 7.9 \\
6480.8846 & -33.5 & 4.6 \\
6485.8048 & -48.7 & 5.5 \\
6507.7828 & -43.8 & 22.7 \\
6533.7565 & 6.9 & 5.1 \\
6552.5484 & 24.4 & 7.8 \\
6556.5333 & 54.7 & 7.2 \\
6563.4964 & 51.5 & 7.6 \\
6569.5599 & 38.7 & 7.1 \\
6577.5286 & 27.4 & 6.6 \\
6581.5111 & 47.6 & 6.4 \\
6790.7998 & -45.5 & 5.0 \\
6805.8595 & -35.1 & 4.8 \\
6822.8399 & 32.9 & 6.7 \\
6838.8336 & 31.1 & 5.4 \\
6855.7274 & 21.0 & 5.1 \\
6879.6707 & 36.1 & 4.7 \\
6882.7526 & 23.1 & 5.0 \\
6892.6339 & 19.3 & 5.9 \\
6906.6214 & 5.8 & 5.9 \\
6915.5634 & -28.3 & 5.0 \\
6922.6643 & -35.6 & 4.8 \\
6931.5519 & -62.2 & 5.2 \\
6936.6461 & -30.1 & 5.5 \\
6939.5186 & -31.1 & 5.3 \\
6943.4909 & -23.7 & 5.4 \\
6949.5001 & -9.0 & 5.0 \\
6951.4918 & -12.2 & 5.3 \\
6957.5240 & 1.9 & 8.4 \\
6966.5438 & 8.8 & 4.2 \\
6975.5073 & 9.0 & 5.4 \\
6983.5068 & 53.2 & 5.4 \\
6991.5867 & 41.5 & 6.6 \\
\hline & & \\
& &
\end{tabular}


Table A.4. FEROS radial velocity variations of HIP 107773 .

\begin{tabular}{lrr}
\hline \hline JD - 2450 000 & $\begin{array}{r}\mathrm{RV} \\
\left(\mathrm{m} \mathrm{s}^{-1}\right)\end{array}$ & $\begin{array}{r}\text { Error } \\
\left(\mathrm{m} \mathrm{s}^{-1}\right)\end{array}$ \\
\hline 5317.8306 & -43.5 & 6.0 \\
5336.9233 & -54.8 & 5.1 \\
5366.9185 & -43.4 & 7.4 \\
5379.8804 & -48.1 & 4.4 \\
5428.7285 & 25.2 & 5.0 \\
5457.6816 & -31.1 & 4.8 \\
5729.8501 & 3.2 & 10.2 \\
5729.8511 & 5.2 & 9.8 \\
5786.8003 & -45.2 & 3.2 \\
5786.8018 & -45.9 & 3.9 \\
5793.7930 & -17.1 & 5.3 \\
5793.7944 & -11.7 & 5.9 \\
6056.8149 & -35.6 & 5.1 \\
6110.8545 & 32.0 & 4.1 \\
6160.7500 & 21.5 & 4.5 \\
6230.5386 & 2.4 & 5.0 \\
6230.5396 & -3.3 & 4.4 \\
6241.5571 & 26.7 & 6.6 \\
6241.5586 & 23.9 & 6.1 \\
6251.5811 & 51.1 & 5.8 \\
6412.8145 & 51.4 & 5.5 \\
6431.8267 & 65.5 & 6.6 \\
6472.8037 & 9.6 & 4.8 \\
6472.8442 & 5.3 & 4.8 \\
6472.8892 & -17.1 & 4.7 \\
6565.6064 & 76.4 & 5.1 \\
6605.6260 & 43.2 & 6.5 \\
\hline & &
\end{tabular}

Table A.5. CHIRON radial velocity variations of HIP 67851.

\begin{tabular}{lrr}
\hline \hline JD - 2450000 & $\begin{array}{r}\mathrm{RV} \\
\left(\mathrm{m} \mathrm{s}^{-1}\right)\end{array}$ & $\begin{array}{r}\text { Error } \\
\left(\mathrm{m} \mathrm{s}^{-1}\right)\end{array}$ \\
\hline 6299.8433 & 32.9 & 4.3 \\
6307.8349 & 51.0 & 4.3 \\
6312.8003 & 57.4 & 4.4 \\
6317.7840 & 62.6 & 4.5 \\
6331.8972 & 103.1 & 4.6 \\
6338.8048 & 118.5 & 4.1 \\
6374.7829 & 15.6 & 4.0 \\
6384.6852 & 17.3 & 4.4 \\
6385.6451 & 24.1 & 4.3 \\
6411.5294 & 54.3 & 4.7 \\
6422.6922 & 92.1 & 4.4 \\
6426.5948 & 91.5 & 4.3 \\
6476.6536 & -7.9 & 3.7 \\
6523.4691 & 62.5 & 4.0 \\
6533.4833 & 48.6 & 5.1 \\
6539.4800 & 40.3 & 9.4 \\
6654.8190 & -60.1 & 6.2 \\
6656.8146 & -47.7 & 5.7 \\
6659.8055 & -35.9 & 4.5 \\
6666.8582 & -12.8 & 5.0 \\
6670.8156 & 13.2 & 4.9 \\
6670.8601 & -0.0 & 5.1 \\
6676.7602 & 19.6 & 5.0 \\
6683.8870 & 40.3 & 5.4 \\
6690.8914 & 44.1 & 6.0 \\
6708.8282 & 27.3 & 4.5 \\
6722.6914 & -22.3 & 4.3 \\
\hline & &
\end{tabular}

Table A.5. continued.

\begin{tabular}{rrr}
\hline \hline JD - 2450 000 & $\begin{array}{r}\text { RV } \\
\left(\mathrm{m} \mathrm{s}^{-1}\right)\end{array}$ & $\begin{array}{r}\text { Error } \\
\left(\mathrm{m} \mathrm{s}^{-1}\right)\end{array}$ \\
\hline 6736.6157 & -68.8 & 5.2 \\
6752.6791 & -45.7 & 4.5 \\
6767.5400 & -18.3 & 4.5 \\
6783.7508 & 7.8 & 5.4 \\
6798.6158 & 1.6 & 5.6 \\
6819.5626 & -69.7 & 6.5 \\
6823.5774 & -64.4 & 5.4 \\
6840.5507 & -61.8 & 4.3 \\
7012.8699 & -108.0 & 6.3 \\
7014.8328 & -100.3 & 5.5 \\
7021.8543 & -76.4 & 5.1 \\
7029.8454 & -51.5 & 4.6 \\
7040.8343 & -6.6 & 5.3 \\
7044.7997 & -12.8 & 5.3 \\
7045.7595 & -12.4 & 5.2 \\
7060.8255 & -7.5 & 5.3 \\
7075.7198 & -54.9 & 5.1 \\
7089.6592 & -80.0 & 4.7 \\
\hline
\end{tabular}

Table A.6. FEROS radial velocity variations of HIP 67851.

\begin{tabular}{lrr}
\hline \hline JD - 2450 000 & $\begin{array}{r}\text { RV } \\
\left(\mathrm{m} \mathrm{s}^{-1}\right)\end{array}$ & $\begin{array}{r}\text { Error } \\
\left(\mathrm{m} \mathrm{s}^{-1}\right)\end{array}$ \\
\hline 3072.8628 & -41.5 & 20.0 \\
3072.8843 & -45.0 & 20.0 \\
5317.6470 & -90.7 & 4.5 \\
5379.6509 & -15.4 & 5.0 \\
5729.6318 & 68.9 & 11.1 \\
5744.6021 & 13.1 & 6.6 \\
6047.6216 & 24.4 & 7.0 \\
6056.6094 & 31.9 & 5.9 \\
6066.6245 & 54.0 & 6.3 \\
6099.6104 & 6.3 & 6.1 \\
6110.5845 & -14.0 & 5.3 \\
6140.6074 & 11.3 & 4.7 \\
6321.7993 & 22.7 & 8.4 \\
6331.7339 & 37.2 & 8.3 \\
6331.8057 & 31.3 & 7.3 \\
6342.7798 & 69.8 & 7.3 \\
6412.5737 & 20.7 & 5.4 \\
6431.5400 & 33.3 & 6.4 \\
6431.5869 & 39.8 & 6.8 \\
6431.6401 & 36.8 & 7.2 \\
6431.6855 & 35.6 & 6.3 \\
6431.7495 & 40.7 & 7.3 \\
6472.6060 & -59.7 & 5.2 \\
6472.6099 & -60.5 & 5.5 \\
6472.6274 & -57.9 & 5.9 \\
6472.6431 & -67.1 & 4.4 \\
6472.6572 & -63.1 & 4.3 \\
6472.7119 & -63.0 & 5.7 \\
\hline & &
\end{tabular}

